\title{
The Relativity Revolution from the Perspective of Historical Epistemology
}

\author{
By Jürgen Renn*
}

\begin{abstract}
This essay analyzes Einstein's relativity revolution as part of a long-term development of knowledge in which the knowledge system of classical physics was reorganized in a process of reflection, described here as a "Copernican process." This process led in 1905 to the introduction of fundamentally new concepts of space, time, matter, and radiation. On the basis of an extensive historical reconstruction, the heuristics of Einstein's creation of the general theory of relativity, completing the relativity revolution, is interpreted as a further transformation of the knowledge of classical physics, starting from conceiving gravitation as a borderline problem between field theory and mechanics. The essay thus provides an answer to the puzzle of how Einstein was able to create a theory capable of accounting for a wide range of phenomena that were discovered only much later.
\end{abstract}

\section{NOVELTIES}

$\mathrm{T}$ he relativity revolution was far from complete in 1905, when Einstein published his pathbreaking paper on the electrodynamics of moving bodies. It started with his reinterpretation of H. A. Lorentz's theory of electromagnetism in what may be called a "Copernican process," in analogy to the transition from the Ptolemaic to the Copernican world system or that from preclassical to classical mechanics. ${ }^{1}$ In such a transition the deductive framework of an old theory is largely preserved while its physical semantics changes, as may be illustrated with the example of Lorentz's auxiliary variable for the local time in a moving reference frame. Originally merely a peripheral aspect of his theory, this auxiliary variable was reinterpreted by Einstein as the time actually measured

* Max Planck Institute for the History of Science, Wilhelmstr. 44, 10117 Berlin, Germany.

For their helpful advice I would like to thank Peter Damerow, Lindy Divarci, and Matthias Schemmel. For his cooperation on many of the issues discussed in this essay, as well as for his challenging critique of the interpretation presented here, I am particularly indebted to Michel Janssen. For his own view see Michel Janssen, "COI Stories: Explanation and Evidence in the History of Science," Perspectives on Science, 2002, 10:457-522.

${ }^{1}$ See Peter Damerow, Gideon Freudenthal, Peter McLaughlin, and Jürgen Renn, Exploring the Limits of Preclassical Mechanics, 2nd ed. (New York: Springer, 2003).

ISIS, 2004, 95:640-648

(C) 2004 by The History of Science Society. All rights reserved.

0021-1753/2004/9504-0006\$10.00 
by clocks in a moving reference system, thus assuming a central role in the new kinematic

framework established by special relativity. Since, according to this framework, physical interactions cannot propagate faster than light, Newton's well-established theory of gravitation, assuming an instantaneous action at a distance, was no longer acceptable after 1905. The relativity revolution was accomplished only when this conflict was resolved ten years later, in November 1915, by Einstein's formulation of the general theory of relativity.

Recent research on the relativity revolution and, in particular, on its second phase, which led to the genesis of general relativity, has profoundly changed not only our image of Einstein but also our understanding of the structure of a scientific revolution. ${ }^{2}$ Einstein no longer appears as the isolated pioneer of twentieth-century physics but, rather, as one who completed classical physics in a way that uprooted its foundations. A scientific revolution now resembles a slow geological process more than a dramatic surge of novelty, since what appeared suddenly to break new ground can instead be seen as having in fact matured over time. Einstein just happened to scale the volcano when it finally erupted.

Yet there seems to be a striking contrast between the first and the last phases of the relativity revolution. The emergence of the special theory of relativity was evidently not an isolated achievement. The virtual simultaneity of the beginning of the relativity revolution with Einstein's other breakthrough discoveries of 1905 indicates that his nonspecialist outlook and, in particular, his youthful pursuit of atomistic ideas enabled him to activate the hidden potentials of highly specialized nineteenth-century physics that others, such as Henri Poincaré, had also exposed.

General relativity, in contrast, might seem to be an example of science without context, created against the common sense of the contemporary scientific establishment. In 1907, rather than giving up Galileo's insight that in a vacuum all bodies fall with the same acceleration, Einstein questioned the barely established kinematic framework of special relativity. In 1912, to the amazement of his colleagues, he abandoned the scalar gravitational potential of Newtonian physics in favor of a sixteen-component object-the metric tensor-with whose mathematics he was barely acquainted. Still, he was able to formulate clear-cut criteria that a gravitational field equation for this monstrous object would have to satisfy. In 1913, however, he discarded generally covariant field equations based on the Riemann tensor, an expression that included second-order derivatives of the metric tensor. He even believed that he had a proof that such field equations had to be ruled out, although in hindsight these were the only acceptable mathematical solution. But in spite of his colleagues' growing skepticism, Einstein maintained his original agenda and returned in late 1915 to field equations based on the Riemann tensor, finally formulating the general theory of relativity with its nonclassical consequences, a theory that essentially has withstood all the later developments of physics and astronomy.

In recent years, all the traditional arguments explaining this dramatic course of events have crumbled under closer scrutiny. Was Einstein's digression caused by his mathematical incompetence? The alleged priority of the mathematician David Hilbert in having formulated the gravitational field equation before Einstein - albeit without giving it a detailed physical interpretation - seemed a strong argument for this interpretation. However, this

\footnotetext{
${ }^{2}$ For extensive documentation of this collaborative research, undertaken jointly with Michel Janssen, John Norton, Tilman Sauer, John Stachel, and others, see Jürgen Renn, ed., The Genesis of General Relativity, 4 vols. (Dordrecht: Springer, forthcoming).
} 
supposed triumph of mathematics over physics moldered under the evidence of the proofs of Hilbert's first paper, which showed that his key insight came only after he had seen Einstein's publication. The deathblow for this line of argument comes from the fact that, in the winter of 1912-1913, Einstein was even able to write down the correct Ansatz for the field equation of general relativity in his "Zurich Notebook," a key document for reconstructing the genesis of general relativity. He later discarded this Ansatz, evidently because he found it unacceptable on physical grounds. ${ }^{3}$

Was Einstein's rambling hence a consequence of physical prejudices that prevented him from recognizing the correct solution even when it was under his nose? The natural candidates for such prejudices - such as the famous "hole argument"- fail on closer inspection to play the evil role ascribed to them. ${ }^{4}$

Among the novelties recently brought to light is the insight that Einstein's investigative pathway was not exactly the solitary enterprise it was traditionally assumed to be. Recent findings have helped to clarify the dependence of Einstein's work on contemporary achievements such as those related to relativistic continuum mechanics and to alternative approaches to the problem of gravitation. ${ }^{5}$ But how do these novel insights actually help us to understand the peculiar character of the second stage of the relativity revolution?

\section{PARADOXES}

The true challenges of the relativity revolution come to light only after the traditional legends have been dispelled. These challenges may be formulated in terms of three paradoxes:

- The paradox of missing knowledge. How was it possible to create a theory, such as that of general relativity, that was capable of accounting for a wide range of phenomena that were discovered only later, in the context of several revolutions in observational astronomy? And whence came the knowledge that granted such stability to a theory that initially did not seem superior to its competitors, since no phenomena were known at the time that could not also be explained by traditional physics?

- The paradox of deceitful heuristics. How could Einstein have formulated the criteria for a gravitational field equation years before he established the solution? How could he establish a heuristic framework that would quickly lead him to a correct mathematical expression and then to the conclusion that it was actually unacceptable-only to bring him back to essentially the same expression three years later?

- The paradox of discontinuous progress. How could general relativity, with its nonclassical consequences such as the dependence of space and time on physical interactions, be the outcome of classical and special relativistic physics - even though such features are incompatible with their conceptual frameworks?

${ }^{3}$ Leo Corry, Jürgen Renn, and John Stachel, "Belated Decision in the Hilbert-Einstein Priority Dispute," Science, 1997, 278:1270-1273; and Renn and Tilman Sauer, "Heuristics and Mathematical Representation in Einstein's Search for a Gravitational Field Equation," in The Expanding Worlds of General Relativity, ed. Hubert Goenner, Renn, Jim Ritter, and Sauer (Einstein Studies, 7) (Boston: Birkhäuser, 1999), pp. 87-125.

${ }^{4}$ For an earlier attempt to identify such a prejudice see John Norton's pathbreaking essay "How Einstein Found His Field Equations: 1912-1915," Historical Studies in the Physical Sciences, 1984, 14:253-316.

${ }^{5}$ See, e.g., John Norton, "Einstein, Nordström, and the Early Demise of Scalar, Lorentz-Covariant Theories of Gravitation," Archive for History of Exact Sciences, 1992, 45:17-94; and Michel Janssen, "Rotation as the Nemesis of Einstein's Entwurf Theory," in Expanding Worlds of General Relativity, ed. Goenner et al. (cit. n. 3), pp. $127-157$. 
These paradoxes can hardly be resolved within a traditional history of ideas. Addressing the challenges they formulate instead requires taking into account dimensions that are usually neglected but are crucial to a historical epistemology of scientific knowledge: the long-term character of knowledge development, the complex architecture of knowledge, and the intricate mechanisms of knowledge dynamics.

\section{LONG-TERM DEVELOPMENT}

An adequate response to the paradox of missing knowledge can be found only when the long-term development of scientific knowledge is taken into account. This development, after all, led to the emergence of a theory whose understanding of how gravity affects motion in terms of space-time structure is closer to Aristotle's concept of natural motion than to Newton's explanation in terms of an anthropomorphic force. The knowledge on which the astonishing stability of general relativity is founded was accumulated long before its creation by centuries of physics, astronomy, and mathematics.

The laws of planetary motion, for instance, including the curious perihelion advance of Mercury that provided the first astronomical touchstone for the new theory, had long been established by extensive observations. Non-Euclidean geometry and the absolute differential calculus - the mathematical language of general relativity - had been developed in the course of the nineteenth century and had even been related to astronomical observations well before the advent of relativity. ${ }^{6}$

The principle that all bodies, independently of their nature, fall with the same acceleration, which was crucial to Einstein's investigative pathway, has been an asset of classical mechanics since Galileo. This principle was the key to Einstein's 1907 insight into the need to go beyond special relativity, since a straightforward generalization of Newton's law to the kinematic framework of special relativity leads to a violation of this principle. The knowledge on which Einstein based his first steps toward a generalization of this framework was also taken from classical physics, as well as from its critical revision by Ernst Mach. In Einstein's famous elevator thought experiment, at the heart of his "principle of equivalence," he established an equivalence relation between physical processes occurring in a system that moves with uniform acceleration and those in a system at rest with a static gravitational field. In this way, he was able to exploit knowledge about accelerated systems when studying gravitational phenomena and, ultimately, to integrate gravitation and inertia in a way similar to the integration of electrical and magnetic fields within special relativity.

Mach had earlier conceived of a structurally similar thought experiment, using a bucket instead of an elevator. He wondered whether the centrifugal forces in a rotating bucket could be ascribed to an interaction between the mass of the water in the bucket and the masses of the distant stars. He also compared an accelerated system-the rotating bucket - with a system at rest in which an interaction between masses, due to the stars revolving around the bucket, accounts for the same physical phenomena as are produced by the inertial forces in the case of the accelerated system. Mach thus effectively provided the blueprint for Einstein's thought experiment.

These knowledge resources may all be considered as part of the shared knowledge

\footnotetext{
${ }^{6}$ See Matthias Schemmel, "An Astronomical Road to General Relativity: The Continuity between Classical and Relativistic Cosmology in the Work of Karl Schwarzschild," in The Genesis of General Relativity, Vol. 3, eds. J. Renn and M. Schemmel. (Dordrecht: Springer, 2005).
} 
available in the early twentieth century to scientists addressing the problem of gravitation. Their attempts to tackle this problem distinguished themselves mainly by the perspective from which these knowledge resources were considered or disregarded. But even the spectrum of individual perspectives may be understood as an aspect of the knowledge system of classical science. This becomes particularly evident when one realizes that the prevailing perspective was that of a specialist working within a subdomain of classical science, while nonspecialist perspectives, being relegated to philosophy or popular science, had come to play a marginal role.

The internal structure of classical physics was largely determined by the emergence of three subdomains with relatively autonomous conceptual foundations: mechanics, thermodynamics, and electrodynamics. At the frontiers of these domains, a class of problems emerged that involved the conceptual foundations of more than one subdomain: the "borderline problems" of classical physics, which generated contradictions between these different frameworks. The electrodynamics of moving bodies stands for the class of borderline problems in which issues of electrodynamic field theory, such as light propagation, were combined with the mechanical question of the role of moving reference systems. This combination gave rise to a clash between the ether model underlying classical electrodynamics and the relativity principle of classical mechanics.

Borderline problems such as these were at the center of Einstein's 1905 papers and catalyzed the transition from classical to modern physics. They gave rise to special relativity, the light quantum hypothesis, and modern statistical physics, respectively. Evidently, their existence was not a matter of Einstein's genius or of favorable local circumstances but the consequence of the long-term development of physics, which also created the conditions for the successful accomplishment of the second phase of the relativity revolution.

After special relativity had elevated the causality requirements implicit in field theory to a universal status - including the requirement that no physical interaction can propagate faster than light — gravitation, traditionally a subject at the core of mechanics, had effectively turned into a borderline problem between mechanics and field theory. As was the case for other borderline problems, its successful solution depended on the shared knowledge resources taken into account. In the case of the creation of special relativity, Einstein's success depended on his combining the heritage of mechanics, embodied in the relativity principle, with the heritage of electrodynamics, embodied in the principle of the constancy of the speed of light. In the case of a relativistic theory of the gravitational field, the combination of the heritage of mechanics represented by the Newtonian theory of the static gravitational field with what was known about dynamic fields from electrodynamics was, however, insufficient to create a new and satisfactory theory-as Einstein's competitors found to their chagrin. There was, in particular, no clue to the properties of dynamic gravitational fields, so that the challenge of building a relativistic field theory of gravitation was comparable to that of developing the entire theory of electromagnetism knowing only Coulomb's law.

It was at this point that Einstein's broad perspective, including his awareness of the philosophical critique of classical mechanics by Mach, allowed him to muster additional resources from classical physics. Einstein exploited the Machian interpretation of the inertial forces in an accelerated reference frame as being due to the interaction of moving masses in order to fill the gap in a field theory of gravitation. By conceiving the inertial forces in accelerated reference frames, such as Newton's rotating bucket, as embodying 
dynamic gravitational fields he managed in fact to anticipate essential properties of the relativistic theory of gravitation he was about to construct, in particular the necessity to generalize the spatiotemporal framework of special relativity, which led to the notion of a curved space-time.

\section{ARCHITECTURE OF KNOWLEDGE}

The answer to the second paradox - how Einstein could have formulated the criteria for a gravitational field equation years before finding the solution-comes from considering the architecture of the shared knowledge resources available to him. These resources were in fact part of a system of knowledge with active components capable of providing heuristic guidance to his research.

The characteristics of Einstein's search suddenly become comprehensible if one realizes that it was guided by a qualitative knowledge representation structure inherited from classical physics: the "mental model" of a field theory as embodied in an exemplary way by Lorentz's electron theory. A mental model in the sense elaborated by cognitive science allows the simulation or anticipation of the behavior of real models despite the lack of full and unequivocal information. Real models serve in turn as external representations of mental models, ensuring their transmission as a part of shared knowledge. According to the "Lorentz model," a source affects its environment in a way that is described by a field equation, while the environment affects the behavior of a probe in a way that is described by an equation of motion. The basic features of the Lorentz model as a model of distant causation may be illustrated by a magnet setting a piece of iron into motion by affecting the state of its environment. The Lorentz model represents a mental model similar to the bucket and the elevator models at the heart of Mach's and Einstein's famous thought experiments.

The internal architecture of a mental model is characterized by terminals open for supplementary knowledge specifying the model. Such additional knowledge is itself structured in a way that may be generically described as a "frame" (mental models are just a specific kind of frame). ${ }^{7}$ A frame, in turn, is an internal knowledge representation structure for objects, operations, or situations that may be externally represented by natural or formal language. Frames have terminals with default settings that represent earlier experiences, thus making it possible to employ frames for interpreting situations even when only incomplete information about these situations is available. Their default settings can be flexibly adjusted if fresh information becomes available, thus making it possible to correct errors without abandoning the interpretation of a situation with the help of a given frame.

The Lorentz model of a field equation has terminals for the source of the field, the field itself, and the differential operator describing how the source generates the field. Einstein's preliminary research on a relativistic theory of gravitation in the years between 1907 and 1912 had established default settings for two of the terminals: the field slot (filled by assuming that the gravitational potential is represented by the metric tensor) and the source slot (filled by the stress-energy tensor of matter, as suggested by relativistic continuum mechanics). For the differential operator describing how the source generates the field, however, Einstein was unable to identify a satisfactory default setting.

\footnotetext{
${ }^{7}$ For the frame concept see Marvin Minsky, The Society of Mind (London: Heinemann, 1987). On the potential of cognitive science and cognitive psychology for the history of science see, e.g., Dedre Gentner and Albert L. Stevens, Mental Models (Hillsdale, N.J.: Erlbaum, 1983); and Peter Damerow, Abstraction and Representation: Essays on the Cultural Revolution of Thinking (Dordrecht: Kluwer, 1996).
} 
Einstein's difficulty did not result from the fact that too little was known but, rather, from the fact that too much knowledge had to be taken into account to formulate a field equation that responded to the understanding of gravitation as a borderline problem of mechanics and field theory. On the one hand, a physically plausible default setting for the differential operator was suggested by knowledge of the Newtonian static gravitational field as well as of the relation between static and dynamic fields in electrodynamic field theory. Constructed in this way, the new theory would automatically be compatible with Newton's theory, thus fulfilling a "correspondence principle." On the other hand, a mathematically plausible way to obtain a default setting for the differential operator was offered by the knowledge about dynamic fields incorporated in Einstein's equivalence principle, which suggested taking generally covariant objects such as the Riemann tensor as the starting point. Constructed in this way, the new theory would automatically fulfill a "generalized relativity principle." Within the knowledge system of classical physics, the Lorentz model was, furthermore, embedded in a network of relations to other frames and mental models; this network served as a control structure for any acceptable implementation of the Lorentz model. In particular, the new theory had to satisfy a conservation principle, generalizing similar principles from classical and special relativistic physics.

In short, Einstein's heuristics was overdetermined by the knowledge of classical physics, which explains why it was so powerful and yet at the same time so fortuitous. The compatibility of the various requirements it imposed could not be established a priori but had to be checked by elaborating a mathematical representation of the Lorentz model, starting from one or the other default setting and shaping it according to the remaining heuristic criteria. Einstein's oscillation between a physical strategy starting from an implementation of the correspondence principle and a mathematical strategy starting from an implementation of the generalized relativity principle can thus be interpreted as realizing alternative and ultimately converging pathways with which to integrate the knowledge of classical physics.

\section{KNOWLEDGE DYNAMICS}

The third paradox, of discontinuous progress, can be resolved only if one takes into account that the development of knowledge does not only consist of enriching a given architecture but also comprises processes of reflection by which this architecture is being transformed. In the case of Einstein's search for the gravitational field equation, the enrichment of the Lorentz model was guided by the relatively stable higher-order structures at the core of his heuristic principles - that is, the principles of correspondence, conservation, equivalence, and generalized relativity.

In addition to the top-down process of assimilation guided by these higher-order structures, Einstein's learning experience was characterized by a process of reflection in the sense of a bottom-up process accommodating these higher-order structures to the outcome of his experiences in implementing his heuristic principles. The interplay between assimilation and accommodation mediated by the mathematical formalism is the crucial process determining the knowledge dynamics leading to the creation of general relativity as a nonclassical theory. Four stages of Einstein's search for the gravitational field equation can be distinguished.

The tinkering phase of fall 1912 is documented in the early pages of Einstein's “Zurich Notebook." It is characterized by his almost complete ignorance of the mathematical opera- 
tions suitable for constructing a field equation for the metric tensor. Nevertheless, reflecting on his first attempts to formulate a field equation that satisfied his heuristic principles, Einstein built up higher-order structures operating on a strategic level that would later guide his systematic implementation of these principles, in particular the physical and the mathematical strategy.

The systematic searching phase from late 1912 to early 1913 is also extensively documented in the "Zurich Notebook." In this phase Einstein, with the help of his mathematician friend Marcel Grossmann, systematically examined candidates according to his heuristic principles, alternating between physical and mathematical strategies. Meanwhile, the relative weight of the heuristic principles kept changing, with the conservation principle emerging as the principal challenge. Paradoxically, the main result of the pursuit of the mathematical strategy was the derivation of an erroneous theory-the so-called Entwurf theory - following the physical strategy. This theory was published in the spring of 1913 .

The consolidation phase is documented by Einstein's publications and correspondence between 1913 and mid 1915. During this phase he elaborated the Entwurf theory, essentially following his earlier heuristics but now working from the perspective of consolidation rather than that of exploration. Paradoxically, however, the main result of the consolidation period was the creation of the presuppositions for a renewed exploration of candidate field equations. Adapting the mathematical strategy to legitimize the Entwurf theory, Einstein found that the resulting mathematical formalism did not single out this theory but reopened the need to examine other candidates, removing, in particular, the difficulty of implementing the conservation principle. Precisely because of the extended network of results meanwhile assembled, this reexamination could now take the form of a reflective reorganization of Einstein's earlier achievements.

The reflection phase, decisive in resolving the paradox of discontinuous progress, is documented by the series of dramatic communications Einstein submitted to the Prussian Academy of Sciences in November 1915. The essence of Einstein's return in the first of these communications to a field equation related to the Riemann tensor consists in reinterpreting results achieved under the spell of the Entwurf theory. As a consequence of this reinterpretation, not only Einstein's original heuristic principles received a new physical interpretation. The Lorentz model itself was transformed, since source, field, and probeits main ingredients - cease to play independent roles, not only because the gravitational field may act as its own source but also because, in general relativity, the equation of motion loses its independent status with respect to the field equation.

The crucial step of the transition from the Entwurf theory, still rooted in classical physics, to the nonclassical theory of general relativity was the shift in the physical interpretation of some aspects of the formalism. ${ }^{8}$ This transition was a Copernican process resembling Einstein's reinterpretation of Lorentz's auxiliary variable for local time as the time measured in a moving reference frame. In passing from the Entwurf theory to general relativity, however, Einstein was his "own Lorentz"- - hence the greater isolation in which the second phase of the relativity revolution took place. In the case of the transition to general relativity, it was the Christoffel symbol, initially only an auxiliary quantity, that assumed a new physical meaning, now representing the gravitational field.

\footnotetext{
${ }^{8}$ See Michel Janssen and Jürgen Renn, "Untying the Knot: How Einstein Found His Way Back to Field Equations Discarded in the Zurich Notebook," in The Genesis of General Relativity, Vol. 2: General Relativity in the Making: Einstein's Zurich Notebook, Pt. 2, ed. Michael Janssen, John Norton, Jürgen Renn, Tilman Sauer, and John Stachel (Dordrecht: Springer, 2005).
} 
A similar effort of physical reinterpretation was absent from Hilbert's contemporary paper on gravitation. This effort did, however, eventually take place in the subsequent reworkings of his original paper, which document a process of equilibration between an individual perspective and shared knowledge resources. In short, even the most ingenious phase of the relativity revolution - the phase of reflection - was, from the point of view of historical epistemology, not the privilege of an outstanding individual but just one aspect of the transformation of a system of knowledge. 